\title{
Seeing through the ground-glass: Do imaging characteristics really matter?
}

\author{
Justin D. Blasberg, MD, ${ }^{\mathrm{a}}$ and Benjamin Resio, $\mathrm{MD}^{\mathrm{b}}$
}

\footnotetext{
From the asection of Thoracic Surgery, Department of Surgery, Yale New Haven Hospital, Yale University, New Haven, Conn; and the ${ }^{\mathrm{b}}$ Department of Surgery, Yale New Haven Hospital, Yale University, New Haven, Conn. This work was funded by the Section of Thoracic Surgery within the Department of Surgery at Yale New Haven Hospital.

Disclosures: Authors have nothing to disclose with regard to commercial support.

Received for publication June 18, 2018; revisions received June 18, 2018; accepted for publication June 21, 2018; available ahead of print Aug 8, 2018.

Address for reprints: Justin D. Blasberg, MD, Yale New Haven Hospital, 330 Cedar St, BB205, New Haven, CT 06520 (E-mail: Justin.blasberg@yale.edu).

J Thorac Cardiovasc Surg 2018;156:1677-8

$0022-5223 / \$ 36.00$

Copyright (C) 2018 by The American Association for Thoracic Surgery

https://doi.org/10.1016/j.jtcvs.2018.06.055
}

Although the preoperative staging of non-small cell lung cancer is founded on imaging characteristics, including tumor size, density, positron-emission tomographic avidity, and regional lymph node involvement, there remains a significant gap in expected 5-year survival for early-stage cancers compared with other solid organ malignancies. Although no single imaging metric represents the "magic bullet" with regard to prognosis, tumor size is classically regarded as the main predictor of risk for locoregional and distant spread. This emphasis is clearly represented in the 8th edition of the lung cancer staging system, which recognizes small differences in tumor size as prognostically significant. Even smaller tumors have the capacity to spread to regional lymph nodes and beyond, however, which is best exemplified by patients with oligometastatic disease and otherwise pathologically normal lymph nodes. Although this is less likely the case for ground-glass nodules, our understanding of tumor biology through preoperative imaging metrics alone is fairly limited, including more recent work on quantitative fludeoxyglucose F 18 (INN Fludeoxyglucose $\left[{ }^{18} \mathrm{~F}\right]$ ) positron-emission tomographic metrics. In their study in this issue of the Journal, Toyokawa and colleagues ${ }^{1}$ provide novel evidence for the association between preoperative computed tomographic characteristics, pathologic features that predict risk for spread, and overall prognosis in patients with early stage non-small cell lung cancer. These findings may prove to be useful in determining preoperatively an individualistic approach to limited resection for subsets of patients with lung cancer.

Previous studies have suggested that limited resection may be inappropriate for patients with high-risk nodules regardless of size, and as a result there have been significant efforts to identify preoperative characteristics that predict

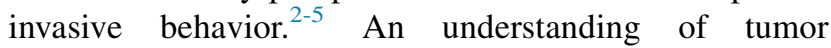
aggressiveness and propensity for spread according to select patients. 6,7



\section{Central Message}

The presence of spread through air spaces is prognostic for survival of early-stage NSCLC. This work describes how features of STAS identified on preoperative CT imaging have clinical significance.

See Article page 1670 .

tumor characteristics and genetic phenotype, however, can only be definitively attained pathologically. Alternatively, preoperative imaging criteria that predict risk for progression have been suggested by studies of ground-glass nodules. Current data supporting limited resection on the basis of preoperative computed tomographic characteristics alone applies, however, only to

Toyokawa and colleagues ${ }^{1}$ retrospectively studied computed tomographic findings, clinicopathologic data, and survival of patients with resected early stage nonsmall cell lung cancer to define cases that were high risk for disease progression after sublobar resection. In their multivariate analysis, the presence of notch (a shallow wavy configuration on the surface of a lesion) and the absence of a ground-glass component on preoperative imaging were independently associated with spread through air space, a pathologic marker of aggressiveness. In addition, these imaging characteristics were associated with decreased recurrence-free and overall survivals. These conclusions are similar to previous work defining the role of sublobar resection for ground-glass nodules ${ }^{8-11}$; however, the analysis of Toyokawa and colleagues ${ }^{1}$ adds to these previous studies by demonstrating that specific combinations of novel imaging metrics are associated with inferior prognosis and that the decision to perform 
sublobar resection should be made with caution in select patients.

Although there may be great promise surrounding the prognostic nature of individual or combinations of imaging features, care should be taken when attempting to generalize these results to patients in the United States. As in all single-institution, retrospective studies, there are inherent limitations of this work that require validation in larger study populations and lung cancer subsets that are phenotypically representative of patients in the United States (particularly given that $68 \%$ of study patients were nonsmokers or light smokers and $50 \%$ had positive epidermal growth factor receptor results). The direct impact of this study's conclusions on clinical decision making should still be considered theoretic, particularly when considering sublobar resection in a patient who might otherwise tolerate lobectomy.

Despite these limitations, the findings of this study suggest an exciting opportunity to use preoperative imaging characteristics to inform risk stratification of pulmonary nodules and thus the extent of pulmonary resection that would be appropriate. As with tumor size, positronemission tomographic avidity, and regional lymph node involvement, defining imaging characteristics that can guide the decision for best available local therapy will likely translate into improved outcomes. Previous work has suggested that the finding of spread through air space should prompt the choice of lobectomy in preference to limited resection, ${ }^{12}$ and the conclusions of Toyokawa and colleagues ${ }^{1}$ provide additional evidence that specific imaging features, such as the finding of notch, are associated with spread through air space. Considering these features during preoperative planning may contribute to identifying and improving survival in high-risk patients. Further multicenter studies on patients with lung cancer in the United States are indicated, however, to validate these findings and the role of sublobar resection for subsets of patients with favorable risk profiles.

\section{References}

1. Toyokawa G, Yamada Y, Tagawa T, Kamitani T, Yamasaki Y, Shimokawa M, et al. Computed tomography features of resected lung adenocarcinomas with spread through air spaces. J Thorac Cardiovasc Surg. 2018;156:1670-6.

2. Chamogeorgakis T, Ieromonachos C, Georgiannakis E, Mallios D. Does lobectomy achieve better survival and recurrence rates than limited pulmonary resection for T1NOM0 non-small cell lung cancer patients? Interact Cardiovasc Thorac Surg. 2009;8:364-72.

3. Dai C, Shen J, Ren Y, Zhong S, Zheng H, He J, et al. Choice of surgical procedure for patients with non-small-cell lung cancer $\leq 1 \mathrm{~cm}$ or $>1$ to $2 \mathrm{~cm}$ among lobectomy, segmentectomy, and wedge resection: a population-based study. J Clin $\mathrm{On}$ col. 2016;34:3175-82.

4. De Zoysa MK, Hamed D, Routledge T, Scarci M. Is limited pulmonary resection equivalent to lobectomy for surgical management of stage I non-small-cell lung cancer? Interact Cardiovasc Thorac Surg. 2012;14:816-20.

5. Khullar OV, Liu Y, Gillespie T, Higgins KA, Ramalingam S, Lipscomb J, et al. Survival after sublobar resection versus lobectomy for clinical stage Ia lung cancer: an analysis from the national cancer data base. J Thorac Oncol. 2015;10: 1625-33.

6. Kakinuma R, Noguchi M, Ashizawa K, Kuriyama K, Maeshima AM, Koizumi N, et al. Natural history of pulmonary subsolid nodules: a prospective multicenter study. J Thorac Oncol. 2016;11:1012-28.

7. Sawada S, amashita N, Sugimoto R, Ueno T, Yamashita M. Long-term outcomes of patients with ground-glass opacities detected using CT scanning. Chest. 2017; 151:308-15.

8. Detterbeck FC. More opaque than clear: reality is always cloaked in shades of gray. Oncology (Williston Park). 2016;30:275-6.

9. Hattori A, Matsunaga T, Takamochi K, Oh S, Suzuki K. Importance of ground glass opacity component in clinical stage Ia radiologic invasive lung cancer. Ann Thorac Surg. 2017;104:313-20.

10. Pedersen JH, Saghir Z, Wille MM, Thomsen LH, Skov BG, Ashraf H. Ground-glass opacity lung nodules in the era of lung cancer CT screening: radiology, pathology, and clinical management. Oncology (Williston Park). 2016;30:266-74.

11. Tsutani Y, Miyata Y, Yamanaka T, Nakayama H, Okumura S, Adachi S, et al. Solid tumors versus mixed tumors with a ground-glass opacity component in patients with clinical stage IA lung adenocarcinoma: prognostic comparison using high-resolution computed tomography findings. J Thorac Cardiovasc Surg. 2013;146:17-23.

12. Kadota K, Nitadori J, Sima CS, Ujiie H, Rizk NP, Jones DR, et al. Tumor spread through air spaces is an important pattern of invasion and impacts the frequency and location of recurrences after limited resection for small stage I lung adenocarcinomas. J Thorac Oncol. 2015;10:806-14. 